Abanico Agroforestal. Enero-Diciembre 2021; 3:1-7. http://dx.doi.org/10.37114/abaagrof/2021.5

Desarrollo Tecnológico. Recibido: 10/02/2021. Aceptado: 17/07/2021. Publicado: 12/08/2021. Clave: e2021-11.

\title{
Cople de camioneta como toma de fuerza para equipos fijos agropecuarios
}

Truck coupling as a power take-off for fixed agricultural equipment

\section{Alejandro Martín-López1, Ayax Jáuregui-Cárdenas', Guadalupe Orozco-Benítez ${ }^{2}$ ID, Isaac Macías-Mendoza ${ }^{1 \text { ID }}$, César Ibarra-Gudiño ${ }^{2}$ ID , Bladimir Peña-Parra ${ }^{2}$ ID , Sergio Martínez-González ${ }^{* 2}$ ID}

\begin{abstract}
${ }^{1}$ Actividad profesional privada. Nayarit, México. ${ }^{2}$ Unidad Académica de Medicina Veterinaria y Zootecnia, Universidad Autónoma de Nayarit. Carretera de cuota Chapalilla-Compostela KM 3.5, Compostela, Nayarit, México. C.P. 63700. E-mail: sam_alex_1@hotmail.com, ayax_goku@hotmail.com, mgorozco63@gmail.com,_isaacus95@gmail.com, cesaroctavio76@hotmail.com, bladiuan73@gmail.com, sergiotepic@hotmail.com
\end{abstract}

Enlace del vídeo HTTPS://WwW.YOUTUBE.COM/WATCH?V=RYGWXIPOYQU

\section{RESUMEN}

La expresión toma de fuerza significa la flecha o flechas que transmiten la potencia al mecanismo de la máquina. Es un eje estriado en un extremo, accionado por un motor. En el presente trabajo se propone un accesorio como toma de fuerza de camioneta para equipos fijos agropecuarios. Esta toma de fuerza consta de una base y una flecha. En la base de dicha flecha tiene una placa redonda que es atornillada directamente a la flecha derecha del eje trasero del vehículo. La propuesta es que los vehículos al fabricarse cuenten con dicho accesorio y tendría un costo aproximado de US\$50.00 (MX\$1000.00). Con este accesorio el empresario agropecuario moverá equipos agropecuarios fijos como molinos forrajeros, mezcladoras de alimento y picadoras. Este diseño conecta la flecha lateral de la camioneta a un equipo fijo agropecuario, de una manera económica y funcional.

Palabras claves: alimentos, maquinaria, tecnología, toma de fuerza.

\section{ABSTRACT}

The expression power take-off is used to indicate the rod or rods that transmit the mechanical power of a machine. It is an axial rod fluted at one end and driven by a motor. In the present work, the power take-off of a light truck is proposed as an accessory for stationary farm equipment. This power take-off consists of a base, and the rod or rods that transmit the mechanical power. At the base of the rods is a round plate that is screwed directly onto the back axis of the vehicle. The proposal is to install this accessory on vehicles at the factory, at an approximated cost of US\$50.00 (MX\$1000.00). With this accessory the farmer can power fixed farming equipment like fodder mills, food mixers and choppers. This design is an economical and functional way to connect the power take-off of the light truck to stationary farm equipment.

Keywords: foods, machinery, technology, power take-off.

\section{INTRODUCCIÓN}

En la agricultura y ganadería como en cualquier industria, la mayoría de los equipos funcionan con una flecha que gira sobre su propio eje; dicha flecha es acoplada a la toma de fuerza de los tractores o de motores estacionarios especialmente diseñados 
para ese fin. Actualmente existen varios diseños de toma de fuerza para altas y bajas revoluciones por minuto, los cuales pueden ser de motores de gasolina, diesel (tractores agrícolas y motores fijos) y eléctricos. Sin embargo, estos equipos por ser especiales son costosos e inalcanzables para el productor agropecuario de nuestro campo, el cual normalmente carece de corriente eléctrica. Se sabe que el productor agrícola y ganadero necesita de equipos que requieren la toma de fuerza, por ejemplo, para las bombas de agua, generadores de energía, molinos y picadoras de forrajes, revolvedoras de alimento, transportadoras de alimento, entre otros. Tomando en cuenta lo anterior, una alternativa de solución del problema de adquisición de motores con toma de fuerza, es la toma de fuerza de una camioneta para equipos fijos agropecuarios, ya que la mayoría de los productores cuentan con una camioneta ya sea de 4, 6 ú 8 cilindros y que además tiene la ventaja de trabajar con bajas y altas revoluciones por minuto.

Se consultaron las bases de datos del Banco Nacional de Patentes del Instituto Mexicano de la propiedad industrial y no se encontró ningún registro en las bases de datos, solicitudes y diseños industriales relacionados con la toma de fuerza de camioneta para equipos fijos agropecuarios, por lo tanto, se ha iniciado el trámite para el registro de patente (Correa y Bergel, 1996; González, 2007).

En los primeros años de vida de la Unión Americana, los agricultores usaban herramientas de mano como la hoz y la guadaña para cosechar el grano. Bueyes lentos tiraban de su arado. Más tarde, y por más de un siglo, usó caballos y mulas para tirar de sus aperos de labranza. La población crecía en las ciudades de los Estados Unidos, requiriéndose más producción del campo para el consumo propio y para exportar a los países que querían comprar. Los nuevos campos y granjas eran mayores; no podían trabajarse a toda su capacidad con los pequeños implementos y fuerza animal. Fue necesaria más y mejor fuerza motriz (Hunt, 1991).

Aproximadamente en 1850, se empezaron a usar algunas máquinas mayores como la segadora y la trilladora de granos, accionadas por una biela del eje del equipo que era jalado por animales (Jonson et al. 1998; Arnal y Laguna, 2000). También se diseñaron aspersoras y segadoras de tracción animal las cuales son movidas por un sistema de biela y manivela del eje, con una caja de engranes que proporciona una multiplicación de giro de 1 a 27 (López et al. 1982a; López et al. 1982b). Del sistema de biela y la caja de engranes parte el proyecto toma de fuerza de la camioneta para equipos fijos agropecuarios. El objetivo del presente desarrollo tecnológico es con la finalidad de que los productores o empresarios agropecuarios con bajos recursos económicos, puedan hacer uso de equipos agropecuarios fijos que requieran toma de fuerza, sin requerir el tractor agrícola o motores eléctricos. 


\section{MATERIAL Y MÉTODOS}

El accesorio consiste en un cople rígido de acero de 4.5 pulgadas de largo y 1.6 pulgadas de diámetro (ver figura 1), que instalado en serie tendría un costo aproximado de US\$50.00 (MX\$1000.00). El cople igual que la toma de fuerza del tractor tiene un estriado estándar para extensiones de flecha agropecuaria y con ranura de seguridad para perno, esto para evitar que se salga la extensión de flecha. La base de dicho cople consta de una placa redonda que es atornillada directamente a la flecha derecha del eje trasero del vehículo. Otro modelo de toma de fuerza podrá atornillarse a los birlos del tambor que sujetan a la llanta (Vaughn, 1988). Es necesario levantar las llantas traseras derechas y asegurar que no tengan movimiento las restantes. Una vez acoplado el equipo forrajero mediante la extensión de flecha a la toma de fuerza (cople), se enciende la camioneta y se decide si se trabajará en bajas o altas revoluciones según el equipo agropecuario a utilizar, entonces se colocará en la caja de cambios de la camioneta la posición adecuada y se procederá a acelerar hasta alcanzar las rpm indicadas por el fabricante del equipo en cuestión. Esta toma de fuerza tiene la ventaja de contar con diferentes velocidades, incluyendo la reversa, que es ventaja para desatascar algunos equipos.

\section{RESULTADOS Y DISCUSIÓN}

Un mecanismo es un dispositivo mecánico que tiene el propósito de transferir el movimiento y/o fuerza de una fuente a una salida (Erdman y Sander, 1998). Y el término cople se refiere a un dispositivo que se utiliza para unir dos ejes en sus extremos con el fin de transmitir potencia. Existen dos tipos generales de coples, rígidos y flexibles. Los coples rígidos se diseñan para unir dos ejes en forma apretada de manera que no sea posible movimiento entre ellos (Mott, 1995). La propuesta es que los vehículos al fabricarse cuenten con dicho accesorio. La toma de fuerza del tractor es un eje, estriado en su extremo, accionado por el motor y destinado a dar movimiento a determinado tipo de máquinas acopladas al tractor (Arnal y Laguna, 2000).

Cuando la flecha es vista desde la parte trasera del tractor, la rotación de la toma de fuerza del tractor es en el sentido de las manecillas del reloj, por lo anterior es necesario que la toma de fuerza de la camioneta sea de la flecha derecha del diferencial, ya que, si conectamos el equipo a la flecha izquierda del diferencial, este último girará al lado izquierdo (Hunt, 1991; Halley, 1990). En la figura 2 se observan equipos fijos agropecuarios conectados a la toma de fuerza de la camioneta. También se podrán conectar equipos de dinamo para generar corriente eléctrica, revolvedoras horizontales o verticales, así como bombas de agua entre otros equipos industriales, siempre y cuando sean fijos (Ortiz-Canavate, 1995). 

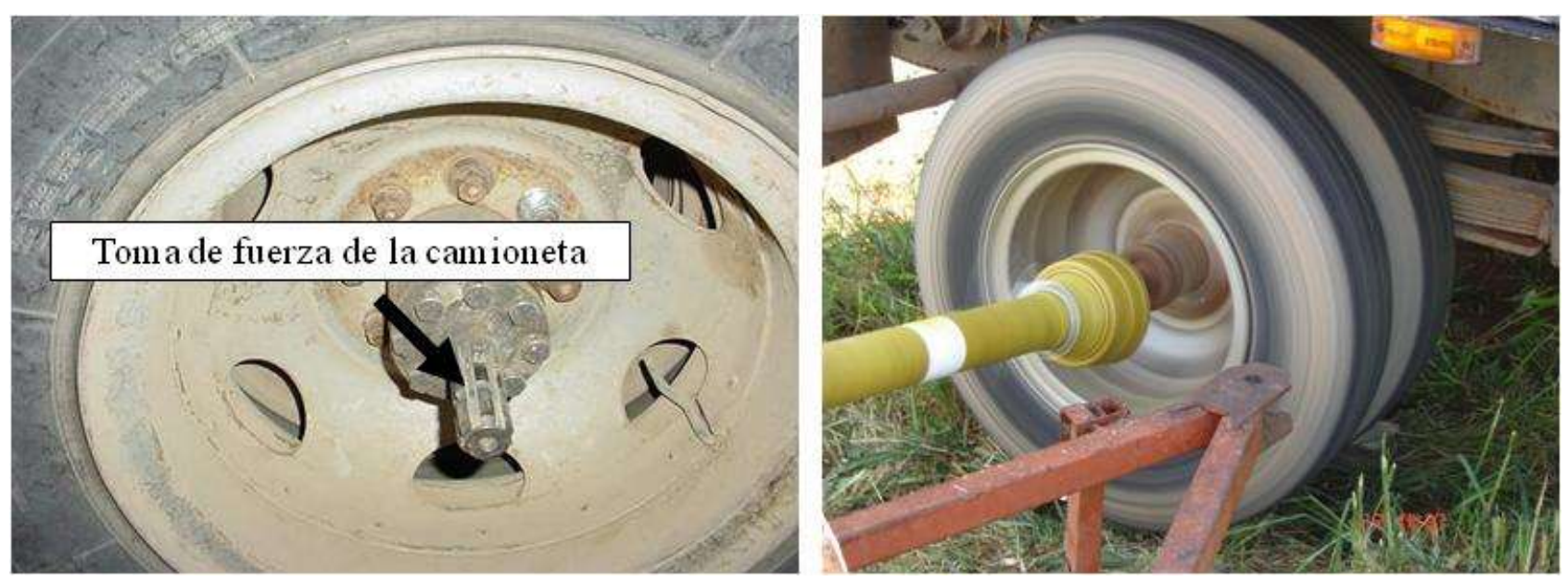

Figura 1. Cople como toma de fuerza
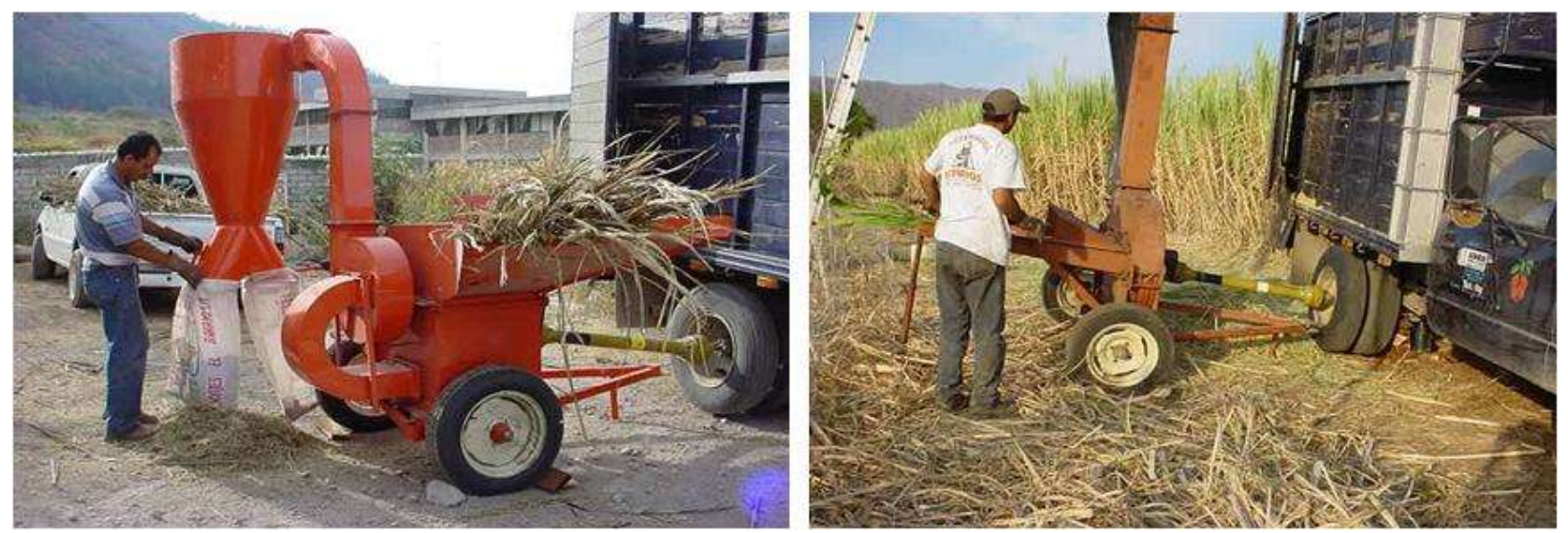

Figura 2. Equipos fijos agropecuarios conectados a la toma de fuerza de la camioneta

La toma de fuerza del tractor tiene por misión dar movimiento y fuerza a los mecanismos internos de algunas máquinas, siempre que estas máquinas estén acondicionadas para recibir movimientos y fuerza. La expresión de "toma de fuerza" significa la flecha o flechas, del tractor que transmiten la potencia al mecanismo de la máquina que lo acompaña (Liljedahl et al. 1984). La toma de fuerza se puede utilizar indistintamente a tractor parado o en marcha $y$, en este último caso, la potencia del motor se reparte entre el accionamiento de la máquina y el movimiento del conjunto tracto-máquina y sobre el terreno. Las medidas del elemento de conexión exterior de la toma de fuerza están normalizadas a nivel internacional, siendo fijos la longitud, el diámetro y el tamaño de estrías, de manera que los fabricantes de máquinas accionadas por la toma de fuerza se adapten a estas medidas y cualquier máquina se puede acoplar a cualquier tractor (Arnal y Laguna, 2000). La velocidad normal de la 
flecha de la toma de fuerza es de aproximadamente $536 \mathrm{rpm}$, aunque ya existe una segunda flecha con 1000 rpm (Hunt, 1991).

Tanto en la camioneta como en el tractor, la toma de fuerza dispone de un sistema de conexión que se efectúa mediante un embrague constituido por un collarín desplazable que, según su posición, hace pasar, o interrumpe, dicho movimiento (Arnal y Laguna, 2000). Las camionetas de 4, 6, y 8 cilindros están capacitadas para trabajar a bajas y altas revoluciones por minuto, con igual cantidad de rpm o más que la toma de fuerza del tractor. Algunas camionetas cuentan con el tacómetro que indica las rpm, según la aceleración del motor. Cuando una camioneta no tiene el tacómetro, es necesario medir el perímetro de la llanta $(\mathrm{P})$ y de acuerdo al kilometraje por hora $(\mathrm{km} / \mathrm{h})$ y dividido entre 60 minutos obtenemos las rpm. Por ejemplo, si una camioneta tiene llantas con 1 metro de perímetro, al acelerarla a $40 \mathrm{~km} / \mathrm{h}$, recorrerá 40000 metros en una hora, o sea 40000 revoluciones por hora, y estas divididas entre 60 minutos, resulta que la flecha lateral del diferencial de la camioneta está girando a $666 \mathrm{rpm}$.

La potencia del motor del tractor, por el contrario que la de camionetas y automóviles, se usa para diferentes objetos. Sin embargo, los tractores no son las unidades adecuadas para muchas empresas pecuarias de tamaño familiar (López et al. 1996). Además, el tractor agrícola es muy lento para moverse, por lo que en la actualidad ya los camiones (con motor de 4, 8 cilindros o mayor capacidad) también son usados para generar corriente eléctrica, aire y para remolcar diferentes equipos agropecuarios.

También existen vehículos con una segunda polea de fuerza, otros tienen el sistema hidráulico. Y lo último, la Mercedes Benz ha fabricado un vehículo con toma de fuerza frontal. Son dos circuitos auxiliares para el accionamiento de elementos como desbrozadoras o barredoras con bombas de regulación de caudal (Mercedes-Benz, 2007).

En cuanto al rendimiento del motor de gasolina comparado con el motor de diesel, todavía no se cuenta con resultados. Sin embargo, en México existen un gran número de equipos agropecuarios fijos que fueron apoyados por Programa Alianza Para el Campo de SAGARPA y muchos de ellos no están trabajando por falta de tractor o corriente eléctrica.

\section{CONCLUSIÓN}

Este diseño conecta la flecha lateral de la camioneta a un equipo fijo agropecuario, de una manera económica y funcional, de tal forma que los productores o empresarios agropecuarios con bajos recursos económicos, podrán hacer uso de equipos agropecuarios fijos que requieran toma de fuerza, sin requerir el tractor agrícola o motores eléctricos. 
AGRADECIMIENTOS

Agradecimientos al Consejo de Ciencia y Tecnología de Nayarit (COCYTEN).

\section{LITERATURA CITADA}

ARNAL APV, Laguna, BA. 2000. Tractores y motores agrícolas. 3 ed. Bilbao, España. Mundiprensa. Pp. 13-374. ISBN 84-7114-645-2.

CORREA CM, Bergel DS. 1996. Patentes y competencia. Buenos Aires, Argentina. Rubinzal-Culzoni. Pp. 7-33. ISBN 95-0727-094-9.

ERDMAN AG, Sandor GN. 1998. Diseño de mecanismos, análisis y síntesis. 3 ed. México DF. Prentice Hall. Pp. 1-30. ISBN 97-8970-170-163-8.

GONZÁLEZ CM. 2007. Reporte de información técnica de patentes de "toma de fuerza de camioneta para equipos fijos agropecuarios". México DF. IMPI (Instituto Mexicano de la Propiedad Industrial). Pp. 78.

HALLEY RJ. 1990. Manual de agricultura y ganadería. México DF. Noriega-Limusa. Pp. 661-699. ISBN 96-8183-618-9.

HUNT D. 1991. Maquinaria agrícola. México DF. Limusa Pp. 271-275. ISBN 968-181308-1.

JOHNSON DM, Harper J, Lawver DE, Buriak P. 1998. Mechanical technology in agriculture. Danville. USA. Interstate Publishers, Inc. Pp. 1-20. ISBN 0-8134-3017-8.

LILJEDAHL JB, Carleton WM, Turnquist PH, Smith DW. 1991. Tractores, diseño $y$ funcionamiento. México DF. Limusa. Pp. 337-360. ISBN 968-18-1703-6.

LÓPEZ GE, Ir JDB, Solís CG, Torres PN, De la Rosa PI. 1996. Organización de operaciones agropecuarias. México DF. Trillas. Pp. 9-20. ISBN 968-24-3741-5.

LÓPEZ GE, Ir JDB, Solís CG, Torres PN, De la Rosa PI. 1982a. Maquinaria para manejo de cultivos. México DF. Trillas. Pp. 67. ISBN 968-24-1156-4.

LÓPEZ GE, Ir JDB, Solís CG, Torres PN, De la Rosa PI. 1982b. Cosechadora de forraje. México DF. Trillas. Pp. 16. ISBN 968-24-1159-9. 
MERCEDES-BENZ. 2007. Toma de fuerza frontal [en línea]. [consultado 4 mayo 2007]. Disponible en el World Wide Web: https://www.mercedes-benztrucks.com/es_ES/brand/campanaespeciales.html

MOTT LR. 1995. Diseño de elementos de máquinas. 2 ed. México DF. Prentice Hall. Pp. 334-363. ISBN 97-0260-812-0.

ORTIZ-CANAVATE J. 1995. Las máquinas agrícolas y su aplicación. 5 ed. México DF. Mundiprensa. Pp. 1-56. ISBN 84-8476-117-7.

VAUGHN RC. 1988. Introducción a la ingeniería industrial. 2 ed. México DF. Reverté. Pp. 1-10. ISBN 84-2912-691-0. 